\title{
AMICal Sat and ATISE: two space missions for auroral monitoring
} \author{
and Mikhail Panasyuk ${ }^{4}$ \\ 1 Univ. Grenoble Alpes, CNRS, IPAG, 38000 Grenoble, France \\ 2 Univ. Grenoble Alpes, CNRS, LiPhy, 38000 Grenoble, France \\ 3 Institute of Engineering, Univ. Grenoble Alpes, Grenoble INP, CSUG, 38000 Grenoble, France \\ 4 Skobeltsyn Institute of Nuclear Physics, Moscow State University, 119991 Moscow, Russia \\ 5 Univ. Grenoble Alpes, IUT1, 38000 Grenoble, France \\ 6 Univ. Grenoble Alpes, Grenoble INP, CNRS, Gipsa-Lab, 38000 Grenoble, France \\ 7 SMC Technological Centre, Zelenograd, 124498 Moscow, Russian Federation \\ 8 ISAE-Supaero, Toulouse, 31400 Toulouse, France \\ 9 ONERA, DOTA, 91120 Palaiseau, France \\ ${ }^{10}$ LATMOS, UPMC, CNRS, 75252 Paris, France
}

Mathieu Barthelemy ${ }^{1,3, *}$, Vladimir Kalegaev ${ }^{4}$, Anne Vialatte ${ }^{1}$, Etienne Le Coarer ${ }^{1,3}$, Erik Kerstel ${ }^{2,3}$, Alexander Basaev ${ }^{7}$, Guillaume Bourdarot ${ }^{1,3}$, Melanie Prugniaux ${ }^{3}$, Thierry Sequies ${ }^{5,3}$, Etienne Rolland, Emmanuelle Aubert ${ }^{3}$, Vincent Grennerat ${ }^{5,3}$, Hacheme Ayasso ${ }^{6,3}$, Arnau Busom Vidal ${ }^{1,3}$, Fabien Apper ${ }^{8}$, Mikhail Stepanov ${ }^{8}$, Benedicte Escudier ${ }^{8}$, Laurence Croize ${ }^{9}$, Frederic Romand ${ }^{9}$, Sebastien Payan ${ }^{10}$,

Received 8 February 2018 / Accepted 14 September 2018

\begin{abstract}
A lack of observable quantities renders it generally difficult to confront models of Space Weather with experimental data and drastically reduces the forecast accuracy. This is especially true for the region of Earth's atmosphere between altitudes of $90 \mathrm{~km}$ and $300 \mathrm{~km}$, which is practically inaccessible, except by means of remote sensing techniques. For this reason auroral emissions are an interesting proxy for the physical processes taking place in this region. This paper describes two future space missions, AMICal Sat and ATISE, that will rely on CubeSats to observe the aurora. These satellites will perform measurements of auroral emissions in order to reconstruct the deposition of particle precipitations in auroral regions. ATISE is a $12 \mathrm{U}$ CubeSat with a spectrometer and imager payloads. The spectrometer is built using the micro-Spectrometer-On-a-Chip ( $\mu$ SPOC) technology. It will work in the 370-900 nm wavelength range and allow for short exposure times of around $1 \mathrm{~s}$. The spectrometer will have six lines of sight. The joint imager is a miniaturized wide-field imager based on the Teledyne-E2V ONYX detector in combination with a large aperture objective. Observation will be done at the limb and will enable reconstruction of the vertical profile of the auroral emissions. ATISE is planned to be launched in mid 2021. AMICal Sat is a 2U CubeSat that will embed the imager of ATISE and will observe the aurora both in limb and nadir configurations. This imager will enable measuring vertical profiles of the emission when observing in a limb configuration similar to that of ATISE. It will map a large part of the night side auroral oval with a resolution of the order of a few $\mathrm{km}$. Both the spectrometer and imager will be calibrated with a photometric precision better than $10 \%$ using the moon as a wide-field, stable and extended source. Ground-based demonstrators of both instruments have been tested in 2017 in Norway and Svalbard. Even though some issues still need to be solved, the first results are very encouraging for the planned future space missions. Data interpretation will be done using the forward Transsolo code, a 1D kinetic code solving the Boltzmann equation along a local vertical and enabling simulation of the thermospheric and ionospheric emissions using precipitation data as input.
\end{abstract}

Keywords: aurora / airglow / spectroscopy / space weather / visible

\footnotetext{
*Corresponding author: mathieu.barthelemy@univ-

grenoble-alpes.fr
} 


\section{Introduction}

Particle precipitations in the upper atmosphere are the main cause of auroral events. They are caused by acceleration processes in Earth's magnetosphere, especially in the auroral acceleration zone. These phenomena are the manifestation of the magnetosphere-ionosphere coupling between high-latitude ionosphere and magnetotail regions (Birn et al., 2012). Each of these regions produces different typologies of auroral emissions. Monitoring particle precipitations in the upper atmosphere is an important aspect of space weather studies since these particles can perturb technological systems and infrastructures on Earth and in space (satellites). These particles, and especially electrons up to $10 \mathrm{keV}$, deposit their energy mainly in the 100-300 km altitude range where auroras occur. Observational data on auroral emissions at these altitudes can give information on particle fluxes at the top of the atmosphere which is very important for understanding of space weather conditions affecting polar Low Earth Orbits (LEO).

The altitude range associated with aurora is too high for balloons, which can reach altitudes up to $50 \mathrm{~km}$, and too low for satellites, which cannot survive for a long period of time at altitudes lower than $300 \mathrm{~km}$. This means that no long-term in situ measurements can be made regarding these particle precipitations. The currently available in situ measurements have been obtained using sounding rockets. Almost all other available data were collected by remote sensing of the ionosphere at these altitudes. Experimental techniques that target optical emissions are particularly powerful since these emissions are mainly related to the excitation processes associated with suprathermal particles.

\section{Choice of observable}

When observing optical emissions several different techniques may be used. We can distinguish: imagery, spectroscopy, polarimetry, as well as some combinations of these (such as, for example, spectropolarimetry).

Polarimetry, especially of the O I red line, has been studied since the first measurements performed by Lilensten et al. (2008). However, in a first stage, our instrumentation will be focused on imagery and spectrometry.

\subsection{Images}

Imagery of the auroral emissions has been practiced for over one century now. The Tromso Geophysical Observatory (TGO) keeps some of the oldest photographic plates $(\sim 1910)$ of auroras taken in Northern Norway (M. G. Johnsen, pers. commun.). In 2017, a large network of all-sky camera surveys the auroral emissions in black-and-white, in color (Red, Green, Blue) or at some specific wavelength, such as that of spectral lines, especially the $\mathrm{O}$ I green line at $557 \mathrm{~nm}$, the O I red line at $630 \mathrm{~nm}$ or the $\mathrm{N}_{2}^{+}$band at $427 \mathrm{~nm}$. These ground based images give some information on the region of precipitation, on the corresponding magnetospheric L-shell, and on the atmospheric and magnetospheric dynamics, as well as composition changes induced by these precipitations. A review of the 0experimental imaging techniques is available in Hecht et al. (2006). Strickland et al. (1989), in particular, were able to fit three parameters (total energetic input, center of the electron energy distribution, and a scaling factor linked to the atomic oxygen profile) from measurements of three auroral lines. However, from the ground, only a small part of the auroral oval can be seen and reconstruction of larger parts of the ovals is extremely difficult with these data. Moreover, cloud coverage prevents a continuous monitoring of the auroral emissions from ground-based stations. In $\mathrm{Ny}$-Ålesund, this cloud coverage can reach $75 \%$ on average over a one-year period (Cisek et al., 2017). From space, some imagers have been able to take pictures of the auroral structure in both limb and nadir configurations. One of the most recent imaging experiments was carried out using the REIMEI satellite (Saito et al., 2011) and especially the MAC optical imager was able to perform auroral images $(64 \times 64$ pixels $)$ at 428558 and $670 \mathrm{~nm}$, at high cadency $(120 \mathrm{~ms})$. These wavelengths correspond to intense emissions of respectively molecular nitrogen ions, atomic oxygen, and neutral nitrogen. In this respect, it is also important to mention the THEMIS network over Canada, which included five satellites and a network of ground magnetometers and 20 ground based cameras to reconstruct the precipitation conditions once every 4 days if weather is clear. Considering the optical part, we propose an architecture with higher time resolution and fewer instruments.

\subsection{Spectrometers}

In addition to the intense lines mentioned above, some molecular emissions extend over a broad wavelength range. Very high spectral resolution enables resolving the individual line profiles providing information on the dynamics of the emitting species. In the Northern hemisphere, we can mention the Ebert Fastie spectrometers installed at the Kjell Henriksen Observatory (KHO) near Longyearbyen in the Svalbard archipelago. Doppler imaging is also able to provide temperature data (Kaeppler et al., 2015). At lower resolution, vibrational structures of the molecular bands are still accessible and it is easier to observe a larger part of the spectra. An example is the Nigel spectrometer in Antarctica at Dome A (Sims et al., 2012), which enables retrieving spectra of the aurora from 300 to $850 \mathrm{~nm}$ at $1.5 \mathrm{~nm}$ resolution; or the auroral spectrograph of the NIPR (National Institute of Polar Research) installed at KHO. A wavelength resolution of $1-2 \mathrm{~nm}$ is sufficient to access the vibrational structures of the main molecular bands.

Considering observations from space, we mention the GLO experiment that flew in the late 80 's on board of the Space Shuttle. Accurate spectra of the airglow have been registered by this instrument (Broadfoot et al., 1997) allowing retrieval of $\mathrm{N}_{2}$ emissions, but only at the relatively low latitudes visited by the space shuttle. If considering auroral regions, the spaceborne spectrometer experiments are mainly dedicated to FUV (Far Ultra-Violet) measurements e.g., the UVI (Ultra-Violet Imager) on board the Polar satellite (Germany et al., 1998) or the TIMED-GUVI (Thermosphere Ionosphere Mesosphere Energetics and Dynamics)-Global Ultraviolet Imager experiment (Christensen et al., 2003). 


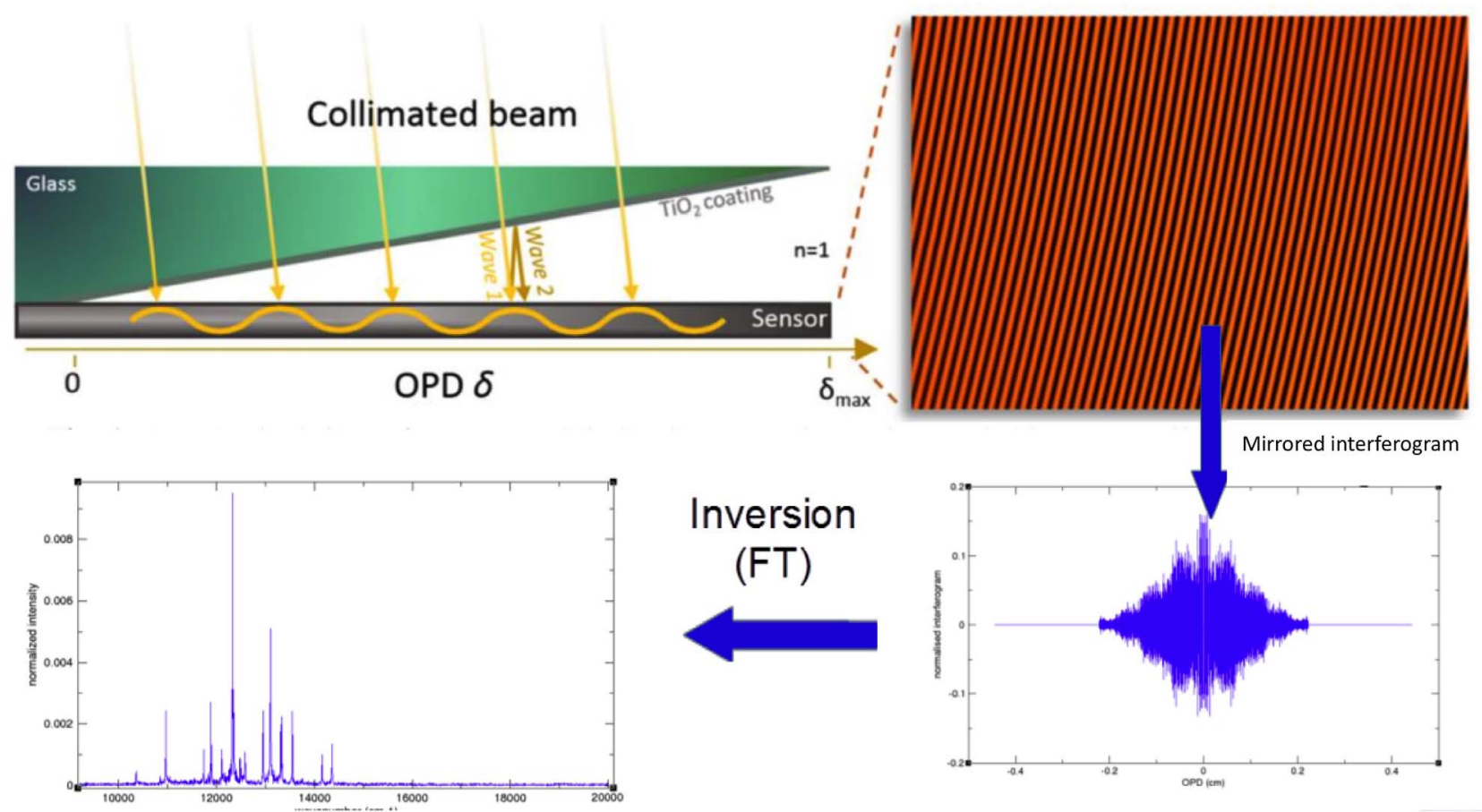

Fig. 1. Schematic representation of the SPOC principle.

\subsection{Retrieval of the vertical profile of the auroral emission}

In terms of information regarding the ionosphere and thermosphere processes, the vertical profile is of great importance since the altitude of the energy deposition depends strongly on the precipitating energy. Using ground-based multi-point instruments, it is possible to reconstruct the deposition altitude by tomography. This is the goal of the ALIS (Auroral Large Imaging System) and MIRACLE (Magnetometers - Ionospheric Radars - All-sky Cameras Large Experiment) networks of cameras installed in northern Scandinavia (Kauristie et al., 2001; Simon Wedlund et al., 2013). In this frame, the Auroral Structure and Kinetic (ASK) instruments suite is also interesting. It enables for some faint emission bands (562.0 nm (ASK1), $732.0 \mathrm{~nm}$, (ASK2), and $777.4 \mathrm{~nm}$ (ASK3)), high frequency $(32 \mathrm{~Hz})$ imaging of the aurora (Tuttle et al., 2014). These methods, however, concern only small atmospheric or spectral regions. It is then more convenient to measure the auroral emissions from space in a limb configuration to enable direct altitude discrimination.

\section{Spectroscopy: the ATISE instrument}

\subsection{The $\mu$ SPOC interferometer}

Considering the relatively faint intensities of auroras and their strong temporal dynamics, Fourier Transform Spectroscopy appears a good technique because of its high light throughput (a.k.a. the Jacquinot advantage) and, provided the measurement is detector noise limited, its multiplexing nature (Fellgett's advantage). The $\mu$ SPOC system developed recently by IPAG and ONERA (Diard et al., 2016) is particularly attractive as it combines the above mentioned advantages with those of a very compact design without moving parts.

The system consists simply of a glass plate directly glued on top of the detectors with a small tilt angle (Fig. 1). Considering its intrinsic reflectivity, the front side of the detector and the glass plate act as the two mirrors of a Fizeau interferometer creating a variable OPD (Optical Path Difference) and fringes localized on the detector. The superposition of the fringes, associated with the incident wavelengths constitutes an interferogram. The spectrum is then retrieved by the Fourier Transform of the mirrored interferogram (Rommeluere et al., 2008). Such a device does not produce an image but an average spectrum of the Field of View (FoV). This means that the scene of interest, i.e. the auroras are not imaged directly on the detectors, but that the optical system projects on the detector an image of the front lens or mirror (field-pupil inversion). This results, for each line of sight, in a uniform illumination of the corresponding Fizeau interferometer.

The major advantage is that a good spectral resolution is obtained while integrating a large optical etendue, resulting in a sensitive spectrometer. The performance of such a spectrometer is mostly limited by the availability of low-noise detectors and the capability of the optical system to uniformly illuminate the detector(s). The size of the instrument is in practice limited by the beam shaping optics before the detector (described below).

\subsection{Science case and instrument requirements}

The characteristics and capabilities of the $\mu$ SPOC have enabled us to design an instrument that will be integrated on 
an economically attractive CubeSat platform (Hevner \& Holemans, 2011). This ATISE instrument will be able to register the vertically-resolved spectral emissions of auroras from the spacecraft in a LEO.

The ATISE spectrometer has been designed with a spectral coverage from 400 to $900 \mathrm{~nm}$ and resolving power of 500 (i.e. $1 \mathrm{~nm}$ at $500 \mathrm{~nm}$ ), to enable the resolution of the vibrational structure of the molecular emission. Resolving the rotational structure, i.e. reaching a resolution of a few $\mathrm{cm}^{-1}$, is not within reach of the $\mu \mathrm{SPOC}$ instrument, considering that different electronic states of the $\mathrm{N}_{2}$ molecule have rotational constants between 1 and $2 \mathrm{~cm}^{-1}$; at least not within the available volume of a CubeSat.

In terms of sensitivity, the objective is to detect a number of more or less faint molecular emissions, including vibronic transitions belonging to, among others, $\mathrm{N}_{2}^{+}, \mathrm{N}_{2}$ and potentially $\mathrm{O}_{2}$. We require that the detection threshold is no higher than $5 \mathrm{R}^{1}$ and that the sensitivity ${ }^{2}$ is at least $1 \mathrm{R}$ for a 1 -second exposure time. Considering the very large range of intensities of auroral emissions, a dynamic range exceeding $10^{3}$ is required.

With a height of $100 \mathrm{~mm}$ and a footprint of less than $200 \mathrm{~mm}$ by $300 \mathrm{~mm}$, the instrument payload then fits in a $6 \mathrm{U}$ CubeSat frame. Including the bus, the entire satellite will adhere to the $12 \mathrm{U}$ CubeSat standard.

In the red and near infrared, $\mathrm{OH}$ exhibits some strong emission lines. These are particularly prominent at altitudes between 80 and $90 \mathrm{~km}$ (Sheese et al., 2014). Since the focus of our instrument is the observation of auroral and airglow emissions in the thermosphere and ionosphere, we choose by default the lowest line of sight at an altitude of $100 \mathrm{~km}$. If the altitude of the satellite is around $650 \mathrm{~km}$, this means that each FoV has a vertical extent of a bit less than $50 \mathrm{~km}$ at the limb configuration $(47.7 \mathrm{~km}$ with a spherical Earth hypothesis). This means that the higher line of sight is between 338 and $386 \mathrm{~km}$, and therewith much higher than the red line emission peak which is found at around $230 \mathrm{~km}$, even if we consider the very large vertical extent of the red line profile (Megan-Gillies et al., 2017). These parameters define the ADCS (Acquisition Determination and Control System) performance required to assure that the spectrometer correctly aims at the same auroral region during the exposure time (of the order of $1 \mathrm{~s}$ ) without risk of including the strong $\mathrm{OH}$ emissions in its FoV. This means that the pointing accuracy and stability need to be better than respectively, $0.1^{\circ}$ and $0.02^{\circ} \mathrm{s}^{-1}$. CubeSat ADCS that meet such accuracy and stability requirements are commercially available in the form of the Blue Canyon X-ACT50 system (with, as of 2018, flight heritage going back to its smaller cousin the XACT-15) or the Hyperion ADCS-400 system. In our case, the ATISE system and platform, including the ADCS, will be realized by the Toulouse University Space Center (CSUT) and commercial ADCS will not be used except as backup solution.

During the nightside of the orbit, observations of the $\mathrm{OH}$ emissions could also be of scientific interest by themself. In this case, a small tilt of the satellite corresponding to one line of

\footnotetext{
${ }^{1} 1 \mathrm{R}$ represents $10^{6} \mathrm{ph} \mathrm{cm}^{-2} \mathrm{~s}^{-1}$ in $4 n$ steradian; See Bake (1974) for more information.

${ }^{2}$ Smallest detectable variation in intensity.
}

sight can be applied to enable their registration. In this case the highest line of sight reaches up to about $338 \mathrm{~km}$.

Since the main goal of the instrument is to both monitor and study the auroral emissions, an almost polar orbit is mandatory. A sun synchronous orbit could then be an interesting choice especially for monitoring the cusp and a part of the auroral oval. However, these orbits have a fixed orbital plane in terms of the local hour. This does not allow studying the physics associated with changing local hour, which is extremely important especially regarding the magnetospherethermosphere coupling. For this reason we choose an orbit with a much lower than $90^{\circ}$, but higher than $70^{\circ}$, inclination, in order to be able to monitor the auroral oval and the cusp. Local hour shift is then slow. At an inclination of $75^{\circ}$, a $12 \mathrm{~h}$ shift takes 60 days at an orbit altitude of $650 \mathrm{~km}$, resulting in a complete view of all local hours every 2 months.

The airglow is much fainter than the auroral emissions. Two observation modes are thus planned with exposure time of $1 \mathrm{~s}$ in auroral regions and $20 \mathrm{~s}$ in airglow regions, with a latency of $1 \mathrm{~s}$ in both modes. The switch between these two modes will be automatic and based on auroral oval simulations based on the $K_{p}$ parameters using the NOAA (National Oceanic and Atmospheric Administration) auroral simulation package OVATION (Newell et al., 2014) or the Sigernes et al. (2011) model. These two simulations give previsions of $K_{p}$ over 3 days, which means that telemetry commands will have to be sent to the satellite at least once every 3 days.

The data rate produced by the experiment will be about 1.5 Gbit/day based on the assumption of six spectra every $2 \mathrm{~s}$ in the auroral region and many fewer in the airglow region (2-4 images per orbit). Since the auroral monitoring sequences for each orbit are linked with $K_{p}$, the data rate increases when magnetic activity increases, reaching $2.5 \mathrm{Gbit} /$ day for $K_{p}=7$.

The planned nominal mission duration of 2 years long will be extended to 5 years if the instrument and the satellite are still working. The altitude of the orbit has been chosen to allow for a 5 years mission. In this case, this will cover almost one half of a solar cycle to allow a monitoring of a large range of solar conditions. Some specific observational modes of operation will be implemented. One will slightly tilt the satellite to lower the lines of sight to enable observation of previously mentioned $\mathrm{OH}$ emissions. This will be performed during nightglow observations.

\subsection{Optical design}

In order to satisfy the mission requirements, the optical design has to meet the following two conditions:

Produce six line of sight detection channels, each covering $1.5^{\circ}$ (horizontal) by $1^{\circ}$ (vertical) FoV. At the limb, each line of sight will cover around $47 \mathrm{~km}$ in altitude with a small gap of $5 \mathrm{~km}$ between each line of sight due to the mounting frame of the six microlenses.

The image of the entrance pupil needs to be projected onto each detector, ensuring a homogeneous illumination of each one of the six detection devices (Gillard et al., 2012; Le Coarer et al., 2014). In order to save space in the instrument, three physical detectors (Pyxalis HDPYX) are used to record the six spatial interferograms associated with the six lines of sight. For this, one line of sight illuminates one half of one physical detector. 


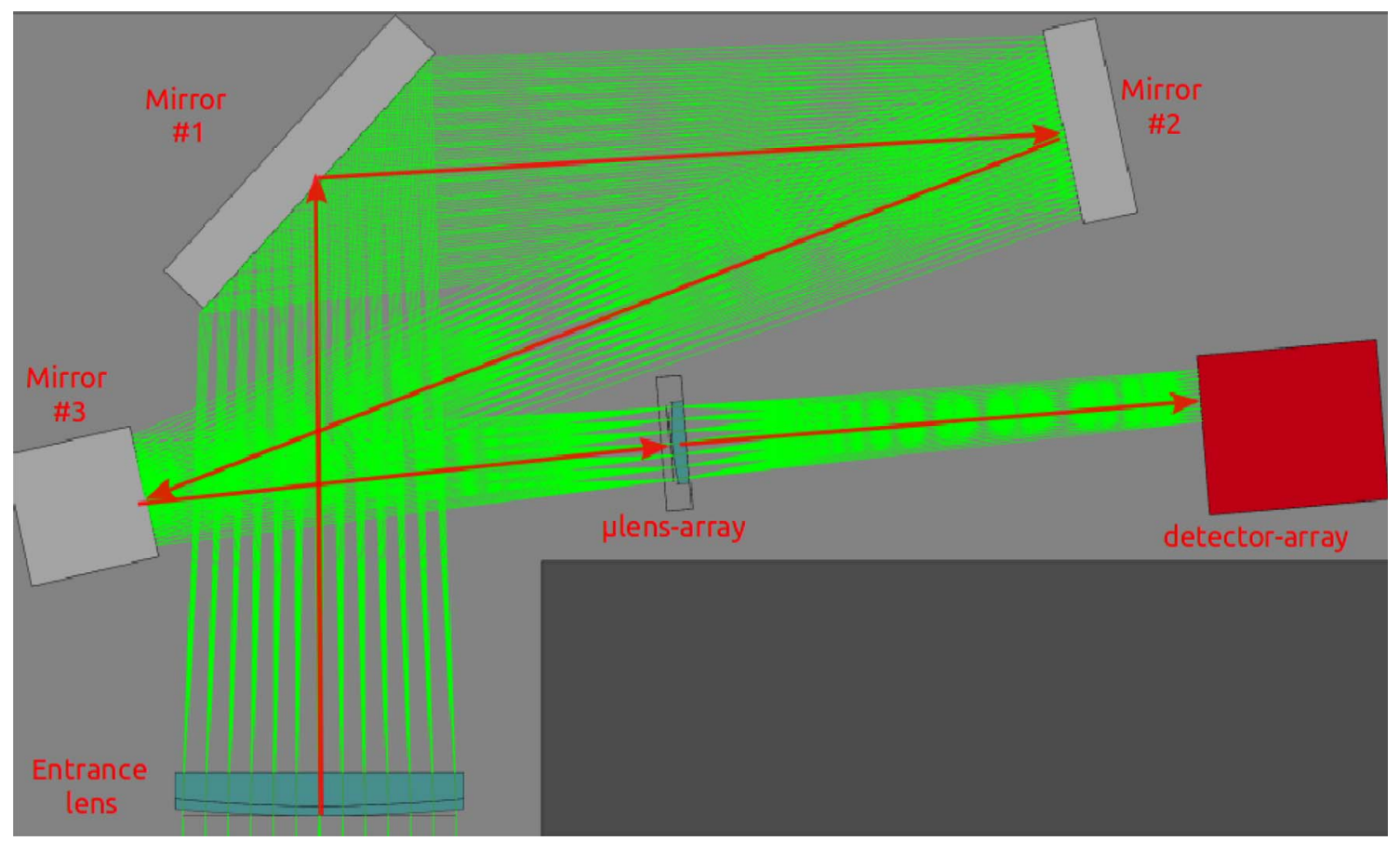

Fig. 2. Optical design of the ATISE instrument. The grey plate represents $6 \mathrm{U}$ size.

The combination of these two conditions requires beam shaping optics with a rather long, folded, optical beam path that consumes about $4 \mathrm{U}$ of the available space. The optical design that was retained, has an entrance lens with a focal distance of about $686 \mathrm{~mm}$, producing an image of the FoV on a micro-lens array consisting of six lenses, placed in the focal plane of the entrance lens (Fig. 2). Subsequently, each micro-lens produces the image of the entrance lens on the corresponding detector. Finally, three plane mirrors fold the optical beam path in the available space.

\subsection{Calibration}

During the mission, the instrument is very likely to experience a performance degradation due to exposure to, among other effects, radiation in space, outgassing, and dust. It is thus necessary to regularly calibrate the spectrometer in order to maintain an absolute photometry of each line and to enable a quantitative comparison to the results of simulations with the kinetic code. Calibration of ground based instruments have been made using point sources like stars (Grubbs et al., 2016). Since the instrument is looking at a spatially extended source, it is more convenient to perform the calibration using extended sources, with very constant photometric flux over the mission duration. The Moon is a unique reference source that meets the above requirements (Teillet et al., 2007). The lunar reflectance is unmatched in its stability, which is better than $10 \mathrm{ppb}$ over one year (Kieffer, 1997). Its radiance is similar to sunlit landmasses, but can be known with much higher precision $(<1 \%)$ and accuracy $(<8 \%)$ (Stone \& Kieffer, 2004). The Moon is frequently used as a photometric calibration source (Wagner et al., 2015), a task that is greatly facilitated by the availability of an accurate dataset and modeling of lunar observations as a function of the phase angle known as ROLO for Robotic Lunar Observatory. The French space agency created a similar tool called POLO for Pleiades Orbital Lunar Observations (Lacherade et al., 2014; Xiong et al., 2014). The precision achievable with the ROLO and POLO models is of the order of $10 \%$ in absolute calibration and $1 \%$ in relative calibration (Goguen et al., 2010). These calibrations cannot be carried out during all phases of the moon. Exact full moon is forbidden due to an increase in the intensity that is poorly simulated by both ROLO and POLO. Periods between first quarter, new Moon and last quarter are also forbidden because too imprecise. Favorable periods are around full moon. One or two calibrations are planned each month.

Moreover, it will also be necessary to calibrate the interferograms on very simple spectra, preferably without molecular emission. This will result in a simple interferogram that can be used to calibrate the OPDs for each of the detector's pixels, which could change with the environmental conditions. The easiest way to achieve this is to rotate the satellite by $90^{\circ}$ with respect to normal operation and to aim all six lines of sight (now horizontal) at one altitude in the upper part of the vertical range. The interferogram will then belong to the spectrum comprised of the three red lines, belonging to the $\mathrm{O}^{1} D-\mathrm{O}^{3} P$ triplet (at 630,636, and $639 \mathrm{~nm}$ ), which exhibit a constant intensity ratio only controlled by the Einstein coefficients. The $639 \mathrm{~nm}$ line is very faint and will normally be hardly visible, if at all.

\section{Imaging: AMICal Sat and ATISE imager}

\subsection{ATISE imager}

It will be fundamental to interpret the spectrometric data, to know what kind of auroral structure the spectrometer is looking at. Since some auroral arcs can be very structured, one of the 
lines of sight can be in and the others out of such an arc. In order to have information on the scene being observed it is thus necessary to use an imager in addition to the spectrometer. Moreover, an imager with a better vertical resolution than the ATISE instrument is a way to interpolate the data within each line of sight of the spectrometer, which will enable increasing the vertical resolution of the entire mission. This requires an on-board intensity calibrated imager with a FoV larger than that of the spectrometer total FoV, i.e. larger than i.e. $6^{\circ} \times 1.5^{\circ}$. The imager data will also have to be calibrated. Since we require an absolute photometry precision of $10 \%$, a Signal-to-Noise-Ratio (SNR) higher than 10 is necessary. To take into account a potential degradation of the instrument during the mission, we impose an initial SNR exceeding 20.

Within the detectors that meet these specifications, we choose the Onyx by Teledyne E2V 1.3 Mpix detector with a pixel size of $10 \mu \mathrm{m}$. This detector is particular in that it is a sparse RGB (Red, Green, Blue) detector with 1 RGB pixel and $15 \mathrm{~B} \& \mathrm{~W}$ (Black and White) pixels out of every 16 pixels.

The required short exposure time will necessitate a large aperture objective and the available volume in the $6 \mathrm{U}$ dedicated for the payload will only allow for a short focal length. An objective between $17 \mathrm{~mm}$ and $35 \mathrm{~mm}$ reaches the requirement if the aperture is $\mathrm{f} / 1.4$, or better still $\mathrm{f} / 0.95$. In these configurations, the FoV of the imager is between $50^{\circ}$ and $26^{\circ}$, which meets our requirement of exceeding the spectrometer FoV. ${ }^{3}$

The images will be compressed on board. Taking images at the same rate as the spectrometer, they will represent the main part of the data volume. The total data volume will reach around 6 Gbit per day which is still acceptable in LEO for $\mathrm{X}$-band data transmissions.

\subsection{AMICal Sat mission}

An imager as described above is by itself an interesting instrument to study auroral phenomena from a space weather point of view, especially if nadir observations are added. This consideration has led us to define a spin-off mission that will embark an auroral imager in a $2 \mathrm{U}$ cubesat. In addition to the limb observation foreseen for the ATISE mission, nadir observation will be performed. This will enable mapping the spatial extend of the oval and distinguishing its internal structure. With the detector and objective as defined for the ATISE mission, a spatial resolution better than $2 \mathrm{~km}$ at an altitude of $110 \mathrm{~km}$ is achievable. The limb observation of the aurora will enable constructing vertical intensity profiles, comparable to those that will be obtained with the ATISE imager. However the ADCS system that will be used in the AMICal $2 \mathrm{U}$ CubeSat, is much more basic. This will necessarily lead to image degradation (blurring) due to both satellite speed and pointing errors. The first source of image blurring is well known, and can be corrected for, provided the SNR is sufficiently high.

AMICal Sat will also test the lunar calibration procedure using the Moon as a reference source that should lead to absolute photometric data with $10 \%$ accuracy and $1 \%$ precision. AMICal Sat is expected to be launched during the first trimester of 2019 for a 1-year mission, extendable to 3 years.

\footnotetext{
3 The final design has converged to a focal length of $23 \mathrm{~mm}$ with an aperture of $\mathrm{f} / 1.4$.
}

Despite the fact that the sun synchronous orbit (SSO) is not totally satisfactory since there is no shift in local hour, this orbit is more easily accessible due to a larger number of launch opportunities. All SSOs with a local hour equal to $12 \mathrm{~h} \pm 4 \mathrm{~h}$ will be acceptable, with a preference for the $10 \mathrm{~h} 30-22 \mathrm{~h} 30$ local hour. The final choice will be determined by the launch opportunities.

AMICal Sat measurements can be synchronized with those provided by the Russian Meteor M2 satellite (Asmus et al., 2014; Kalegaev et al., 2018). The orbit of Meteor M2 is an $\mathrm{SSO}$ at an altitude of about $800 \mathrm{~km}$ altitude. It has on-board meteorological instruments, including detectors developed at MSU. One of the detectors measures fluxes of $\mathrm{keV}$ charged particles. Comparison of AMICal Sat and Meteor M2 measurements in magneto-conjugate regions will enable obtaining more information on mechanisms of auroral particle precipitations.

AMICal Sat will use the S-band for data transmission, meaning it will necessarily take fewer pictures than ATISE. As for ATISE, recording will be limited to the auroral oval only. The extent of the auroral oval will be estimated based on $K_{p}$ forecast values. To limit the number of pictures and therewith the data rate, images registration frequency will be lower than in the ATISE case. A superposition of $1 / 3$ of each photo, regarding the previous one, is then planned in nadir mode. This will allow to both set positioning of the satellite during images recording and to limit the number of images. In limb mode, one picture every $30 \mathrm{~s}$ is planned instead of one every $2 \mathrm{~s}$. Data production is then limited to around 250 Mbits per day.

\section{ATISE ground-based demonstration: 2017 campaign in Skibotn}

\subsection{Observation condition}

Since the ATISE concept has never been tested for auroral emissions, a ground-based demonstrator was built and tested in Skibotn (Norway) at the end of February, beginning of March, 2017. The optical design was identical to that of the space payload design, except for the fact that only one half detector was active meaning that only one line of sight was registered. Also, the detector proximity electronics were different from those of the future satellite electronics. The instrument was installed in the Skibotn observatory cupola $\left(20^{\circ} 21^{\prime} 54^{\prime \prime} \mathrm{E}\right.$, $69^{\circ} 20^{\prime} 54^{\prime \prime} \mathrm{N}$ ). During the first 3 days of the campaign, it looked toward the West with an elevation angle of $30^{\circ}$, while the remaining time it looked toward the East (same elevation angle).

During the first two observation nights, the exposure time was $4 \mathrm{~s}$ as the optical system was not totally optimized. The exposure time was subsequently reduced to $2 \mathrm{~s}$.

\subsection{Results}

Figure 3 shows a spectrogram of data collected during almost $10 \mathrm{~h}$ on March 1 . Wavelength is shown on the vertical axis, while time is on the horizontal axis. The intensity of the spectra is color coded. The green line emission is easily recognizable in all spectra recorded throughout the night. A large 


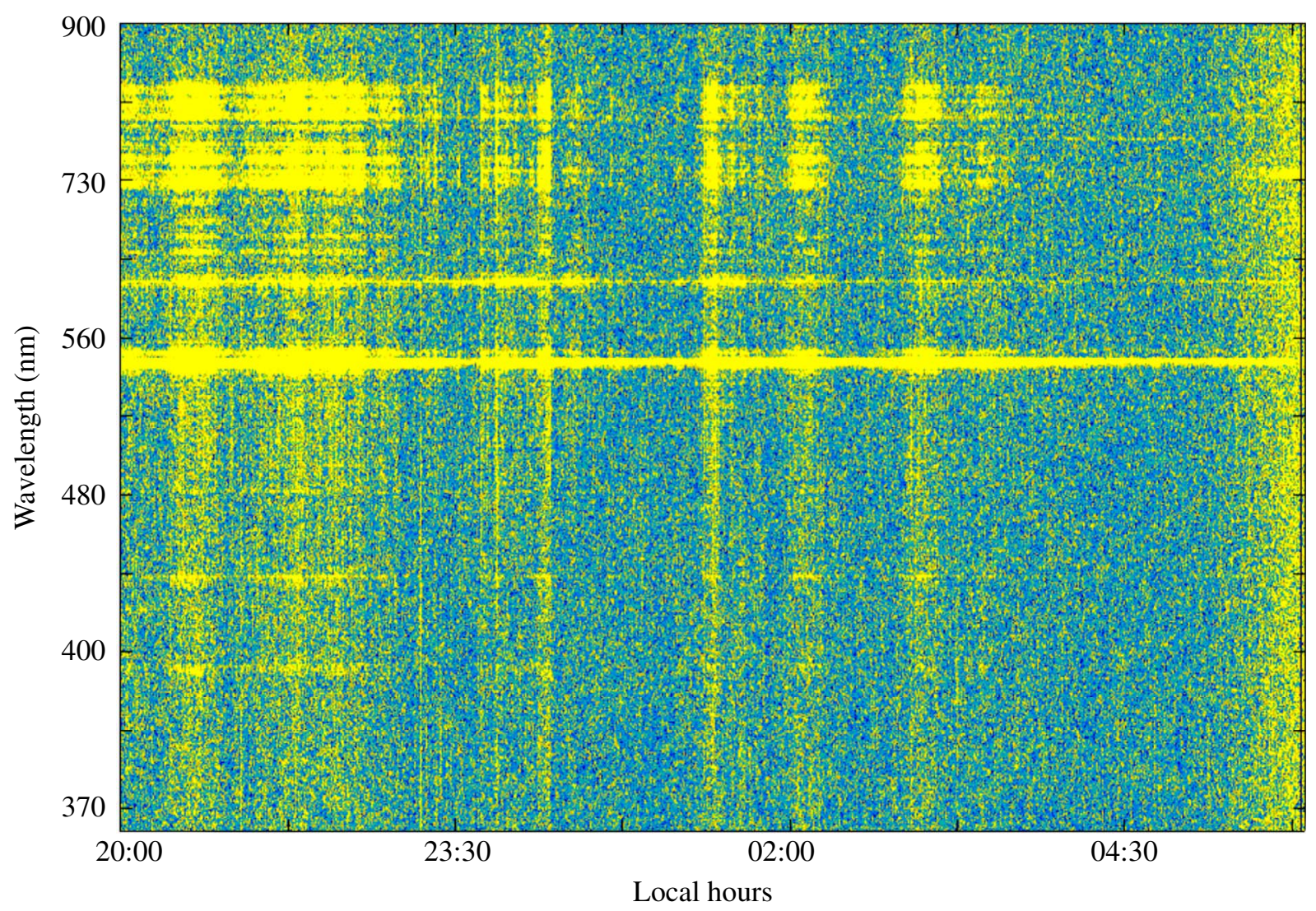

Fig. 3. Spectrogram of a series of spectra collected during the almost 10 hours of observation in March 2017. Wavelength (in nm) is on the vertical axis and the local time is on the horizontal axis. The red and green lines are easily recognizable at respectively 557 and $630 \mathrm{~nm}$. The two $\mathrm{N}^{+}$lines at 391 and $428 \mathrm{~nm}$ are faint but recognizable. The $\mathrm{N}_{2}$ first positive and $\mathrm{N}+$ Meinel features are present in the red part of the spectra (see also Fig. 4). The dawn at the end of night is also recognizable at the right of the figure. The wavelength scale of the figure is approximate and does not correspond exactly to the fit mentioned in the text. Some strong enhancements of the total intensity are visible. These enhancements seem to concern all wavelengths. This appears to be some combination of different effects: A strong enhancement of all line intensities that is real and due to greater auroral activity. However, since the intensity scale is the same for the entire night, the noise becomes much larger when the total number of photons increases, giving the impression of continuous emissions. This is of course a fake process exacerbated by the strong noise experienced during the ground based demonstration. A third effect is due to the Fourier transform process, despite the apodisation windows used. These data processing effects will be resolved by ground based calibrations.

group of intense lines is present in the red, including the oxygen red line $(630 \mathrm{~nm})$ and the line at $844.6 \mathrm{~nm}$ corresponding to the atomic oxygen $3 p^{3} P-3 s^{3} S$ transition. In the blue and near UV, the most visible features are the $N_{2}^{+}$bands of the first negative system $\left(B^{2} \Sigma_{u}^{+}-X^{2} \Sigma_{g}^{+}\right)$at $391(0-0)$ and $427 \mathrm{~nm}$ $(0-1)$. To facilitate the identification of the other spectral features, a wavelength calibration was performed by fitting a polynomial to the positions of the 427.8, 577.7, 630.0 and $844.6 \mathrm{~nm}$ lines. We used a third order polynomial with the following parameters:

$$
\begin{aligned}
P(x)= & -3.747 \times 10^{-7} x^{3}-3.923 \times 10^{-4} x^{2} \\
& +2.067 \times 10^{1} x+9.168 \times 10^{3}
\end{aligned}
$$

With this fit the identification of the principal lines is quite satisfactory as shown in Figure 4 for the red part of the spectra. Lines from both the $\mathrm{N}_{2}$ first positive band and the $\mathrm{N}_{2}^{+}$Meinel band are correctly identified. While emissions above $700 \mathrm{~nm}$ are clearly present and easily assigned, it is more difficult to assign the features between 630 and $700 \mathrm{~nm}$ where lines of the $\mathrm{N}_{2}$ first positive bands should be present.

One important point is the stability of the spectrometer in terms of sensitivity and wavelength calibration. We have several means to characterize these. Firstly, some lines such as the $630-\mathrm{nm}$ and $636-\mathrm{nm}$ lines of O I or the 427-nm and 391-nm lines of $\mathrm{N}^{+}$must have a constant intensity ratio since they share the same upper level. Secondly, we can compare our spectra with those obtained by another instrument, the "premier cru" spectropolarimeter that was operated during the same time frame and on the same line of sight.

In order to achieve this, the intensities of each line must be determined. For the prototype of ATISE, we can fit Gaussian profiles to the visible features and use the fitted line areas, numerically integrate the line profiles to obtain their intensity, or use the center line intensity for each feature (effectively assuming that all lines have the same width). This hypothesis and the fact that some significant overlaps occur make the latter 


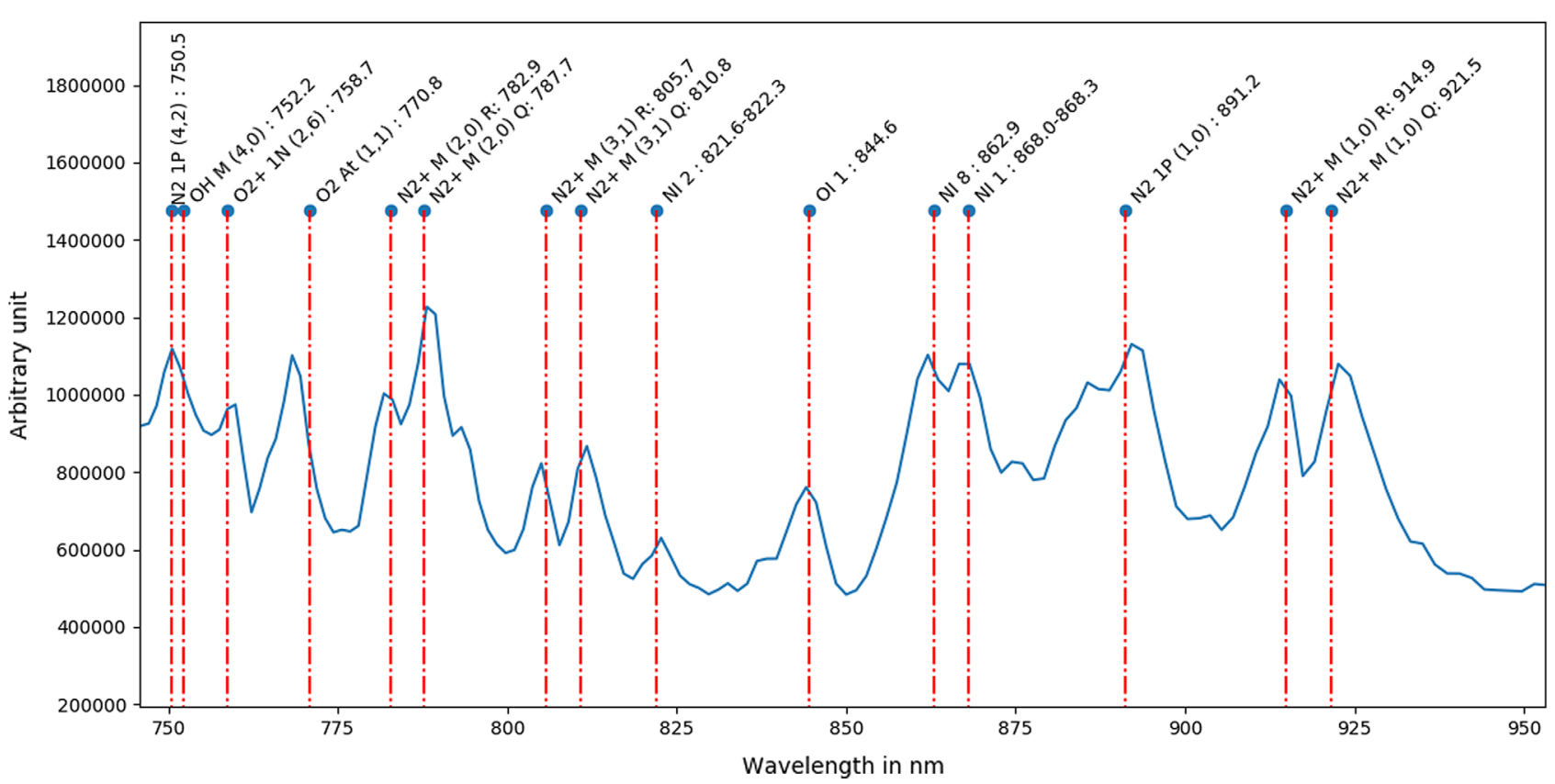

Fig. 4. Section of the spectrum between $750 \mathrm{~nm}$ and $930 \mathrm{~nm}$ averaged over one night (March 1, 2017). Identification of the lines is given at the top of the figure.

two options problematic. We therefore proceed with a fit of multiple Gaussian profiles to the spectra.

Following the NIST data and Baluja \& Zeippen (1988), the ratio $I_{630} / I_{636}$ should be slightly larger than 3 . The ratio is, however, clearly higher for the ATISE data. It reaches 4.2 for the data of March 1 and 3.7 for the data of March 4. The $636 \mathrm{~nm}$ line is not detectable in the data of March 2. As for the $427-\mathrm{nm}$ to $391-\mathrm{nm}$ line intensity ratio, the theoretical branching ratio (Torr \& Torr, 1982) is 0.35 . It is clear that in the ATISE data this ratio is not respected. This is however easily explained since the sensitivity of the detector is falling strongly between $427 \mathrm{~nm}$ and $391 \mathrm{~nm}$, mostly due to the varying reflectivity of the detector surface. This reflectivity is higher in the red resulting in a higher sensitivity in that part of the spectrum. Moreover, small irregularities at the surface of the detector, due to the passivation layer, can perturb the interferograms and this may well explain the observed discrepancy between previously determined line intensities and those measured by the ATISE demonstrator. This will be corrected using two different strategies:

- Instead of using frontside illuminated detectors, ATISE will use backside illuminated detectors. This solution will reduce the irregularities mentioned above by allowing a thinner passivation layer and then remove their effect from the interferograms. This is also expected to partially solve the drastic reduction of the sensitivity in the blue and nearUV regions.

- An additional correction will be added to the data processing by calibrating the spectrometer on the ground using spectral calibration lamps with known intensity ratios.

Regarding the requirements in terms of sensitivity, it is clear that the $5 \mathrm{R}$ detection sensitivity threshold is not reached.
The noise is larger than expected. An analysis after the campaign showed that a grounding error (ground loop) in the proximity electronics increased the noise level. This error has been corrected and will thus not affect the space version of these electronics. The ground loop error does explain why it was impossible to record good spectra with a 1-second exposure setting during the test campaign.

The test campaign thus clearly revealed some problems with the spectrometer that need to be addressed. The electronics problem has been identified and corrected after the campaign, whereas calibration issues with the spectrometer will be corrected by using backside illuminated detectors and proper data processing procedures.

In general, the tests show that the ATISE instrument is able to record spectra with a SNR that is sufficient for the auroral observations. The instrument was also shown to be sufficiently stable in terms of both sensitivity and wavelength calibration to enable identification of all major features in the recorded spectra.

\section{Coupling with the Transsolo code for space weather applications}

After solving the previously mentioned issues, fully calibrated spectra of the aurora will allow retrieving some space weather parameters through modeling of notably the particle precipitation fluxes. The simulation results can also be compared to those measured directly at $800 \mathrm{~km}$ altitude by the Meteor-M2 satellite.

\subsection{Interpretation of the data}

Altitude resolved spectra can be interpreted using kinetic codes that calculate the light emissions based on an 


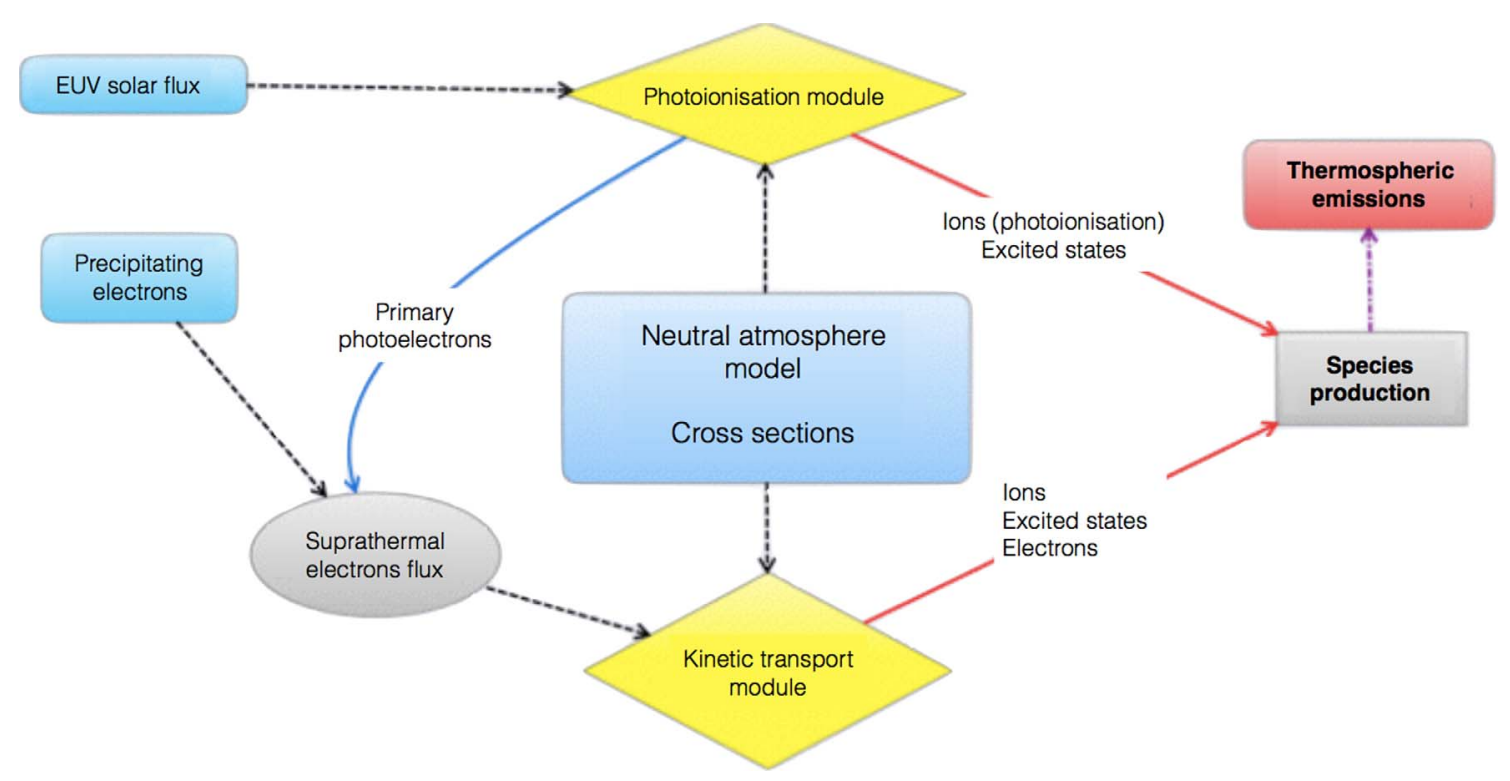

Fig. 5. Schematic view of the Transsolo simulation flow. Inputs are in blue and output in red.

atmospheric model and the energetic inputs like solar UV flux and particle precipitations. Several of these models exist, some with bi-directional flux calculation (up and down fluxes), others with multi stream calculations. The model to be used with the ATISE and AMICal data will be the Transsolo code (Lummerzheim \& Lilensten, 1994; Lilensten \& Blelly, 2002) developed over the last 25 years to calculate the vertical transport of the particles through the thermosphere and the associated light emissions. The code has recently been used to calculate the NO emissions in auroral regions (Vialatte et al., 2017). The Transsolo code is a multi-stream kinetic code that allows from inputs such as the solar EUV flux and the electron precipitation to calculate the emissions. It is a $1 \mathrm{D}$ code meaning that all calculations are done along an axis which is often the magnetic field line. A schematic overview of the Transsolo flow is presented in Figure 5. This code takes the atmospheric model as an input. In our case, two models are useable: NLRMSISE 2000 (Picone et al., 2002) or IRI (Bilitza et al., 2012).

\subsection{Spectroscopic data}

It is first clear that the recorded intensities for each line of sight do not only comprise the intensities of the auroral emissions at the tangent point of the line of sight, but actually integrate all emissions, also those from higher altitude atmospheric layers since the satellite will fly at $650 \mathrm{~km}$. This means that some O I red line emissions will be visible, even for the lowest line of sight. Concerning the interpretation of the data with the Transsolo code this means that several simulations corresponding to different geographical positions will be required, especially for the lowest line of sight. Since the Transsolo code is a $1 \mathrm{D}$ code, the only way to accomplish this is by running it several times using the hypothesis of negligible horizontal transport.

The advantage of a large band spectrometer is to enlarge the number of emission lines and bands accessible; enabling a more complete characterization of the system. It will, however, be necessary to limit the number of explored parameters, compared to the number of input parameters of the code. It is clear that priority will be given to the energy distribution and total flux of the precipitating particles, which is similar to the parameters explored by Strickland et al. (1989). Since we use independent atmospheric models (MSIS or IRI), we will a priori avoid to use the scaling factor linked to the atomic oxygen profile. However, considering only one particle distribution is often not sufficient to characterize the particle precipitation, especially since the magnetospheric sources can be multiple. The inversion procedure will then allow to superimpose several distributions. The shape of the distribution will also be investigated using a limited number of pre-determined shapes (Gaussian, Maxwellian, etc.). In a first stage, by using multiple direct runs of the code, a positioning of the measured results in the input parameter space will be feasible, and will allow for the interpretation of the data in a reasonable amount of time. However, a study of a proper inversion procedure of the data is ongoing and will be finalized before the ATISE satellite launch in 2021. Strategies like those used by Simon Wedlund et al. (2013) based on the multiplicative algebraic reconstruction technique or the maximum entropy method could be adapted since the forward model is similar. A first version will be tested on an AMICal Sat dataset.

\subsection{Imaging data}

The limb image data by themselves will be interesting for space weather studies, but the amount of information that they can provide is limited. In a first processing step, only the total intensities of the vertical profile will be used. In a second step, the sparse RGB information will be processed. This means that if interpretation of the limb data of AMICal Sat will be carried out using the Transsolo code, it is likely that the solution is ill-determined, especially when not using the RGB information in the first processing step. However, since Space Weather is a 


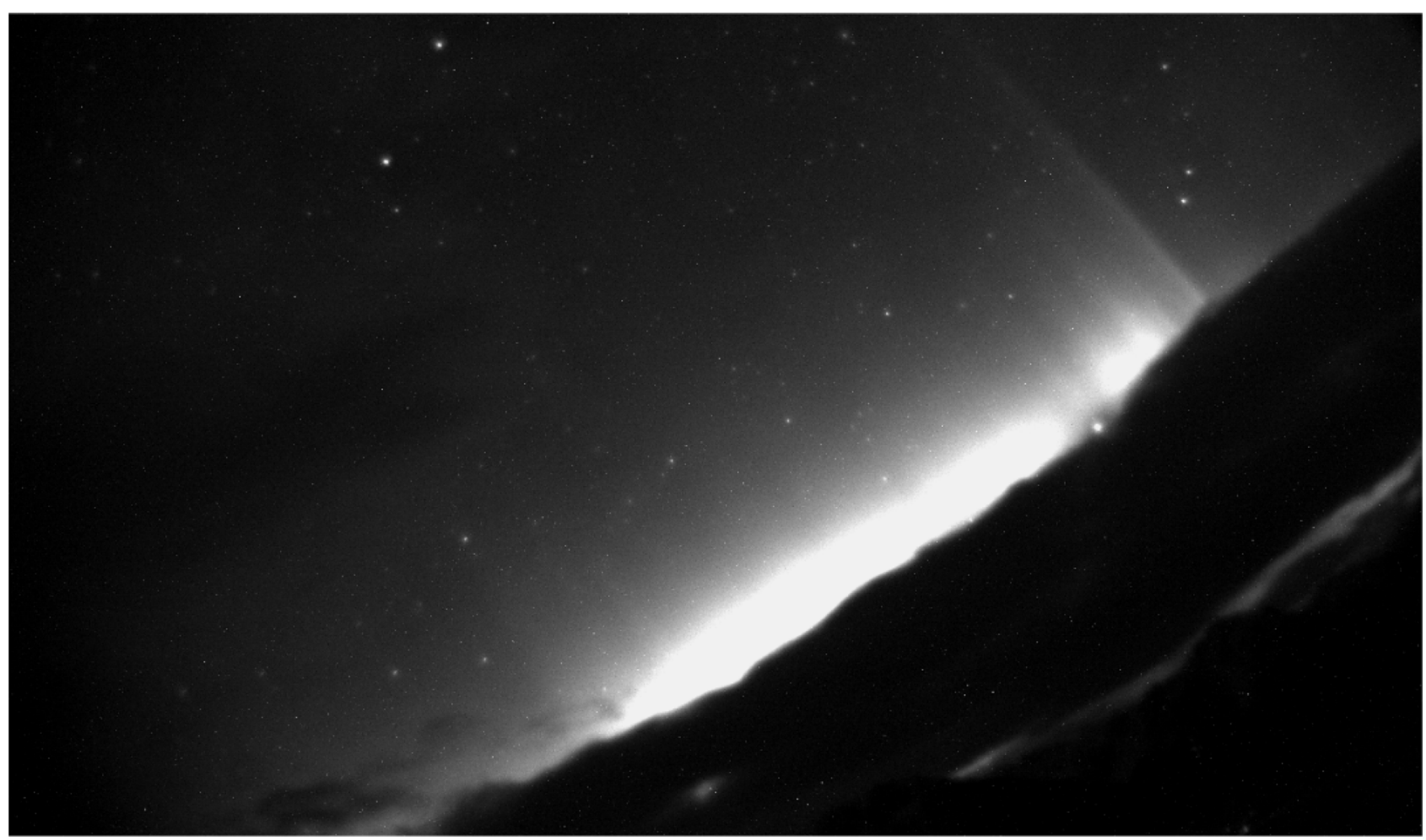

Fig. 6. Aurora image taken with the demokit of the ONYX detector during the Ny-Ålesund Nov 2017 campaign. The exposure time is $0.82 \mathrm{~s}$, the analog gain is $\times 6$. The instrument was inclined at about $45^{\circ}$. The aurora was faint, barely visible with naked eyes.

multi-instrument and multi-measurement science, some coordinated measurements will be possible with some ground based instruments such as the EISCAT radars, which give the state of the ionosphere along the radar line of sight, or with optical instruments such as the ALIS cameras.

For nadir imaging, observation of the oval is by itself extremely interesting since it gives a direct mapping of the magnetic field lines that affect the particle precipitations. This will be extremely useful for the magnetosphere-ionosphere coupling study, giving a more precise idea of the location of radiation belt regions and of subsequent magnetosphere processes. Small scale structure of the size of a few kilometers in a short observation time will be visible, and will allow for a much better comprehension of the dynamics of the particle precipitations.

A black and white prototype of the imager have been tested in November 2017, in Ny-Ålesund (Spitzberg, Norway). An objective with a focal length $f=17 \mathrm{~mm}$ and a numerical aperture of $\mathrm{f} / 1.4$ has been fitted to a demo-kit of the Teledyne-E2V ONYX detector. An image taken during this campaign is shown in Figure 6. The main conclusion of this campaign is that the imager configuration is sensitive enough for faint auroras with exposure times of the order of $1 \mathrm{~s}$, thus validating the instrument concept.

\section{Conclusions}

As stated in the previous paragraphs, the demonstration of the ATISE prototype is convincing, even though not all issues regarding the space mission requirements are resolved and some improvements in both hardware (noise reduction, backside illumination) and data processing are still needed. The ATISE and AMICalSat missions are expected to deliver a very large set of data for a large variety of space weather studies. Vertical profiles will enable the reconstruction of the altitude at which the dominant emissions take place, and constraints on the particle precipitation up to few $\mathrm{keV}$. These particles are not the most energetic ones, but by their abundance, they play a major role in the thermosphere-ionosphere dynamics. The fast time response of the instruments will enable reconstructing dynamics with good accuracy. Before the launch of the satellites, several additional ground based campaigns will be performed with both the imagers and the spectrometer. The ATISE spectrometer will be tested again during a campaign planned for the 2019 winter. Data on particle precipitations obtained during the ATISE flight campaign will be compared with measurements of low-energy electron fluxes made by Meteor-M2 or similar LEO satellites. We also plan to make sure that the ATISE mission will coincide with measurement campaigns of particle instruments, such as the Dynagrad developed by ONERA, or similar auroral electron detectors included in the "UniverSat Socrat" mission currently developed by the Moscow State University (Panasyuk et al., 2017). The two satellites will be included in this large multisatellite mission dedicated to Space Situational Awareness (SSA).

As to the future prospects of ATISE, we mention that it would be extremely interesting to fly multiple ATISE satellites 
in a constellation in order to increase both spatial and temporal coverage, as would be the development of a hyperspectral version of the ATISE spectrometer.

Acknowledgements. The AMICal Sat and ATISE missions are being developed by the CSUG and MSU/SINP, in collaboration with the CSUT and SMC TC. CSUG is funded by Air Liquide Advance Technologies, Teledyne E2 V, Nicomatic, Sofradir and Gorgy Timing through the UGA Foundation. The MSU/SINP team was supported by RFBR grant 16-05-00760. Educational aspects have been partly funded by the IDEX in Grenoble. The ATISE prototype has been funded by the Auvergne Rhone Alpes region. This work was partly supported by the Programme National PNST of CNRS/INSU co-funded by CNES and by CNES. The editor thanks Johan De Keyser and an anonymous referee for their assistance in evaluating this paper.

\section{References}

Asmus VV, Zagrebaev VA, Makridenko LA, Milekhin OE, Solov'ev VI, Uspenskii AB, Frolov AV, Khailov MN. 2014. Meteorological satellites based on Meteor-M polar orbiting platform. Russ Meteorol Hydrol 39(12): 787-794. DOI: 10.3103/S1068373914120012.

Baker DJ. 1974. Rayleigh, the unit for light radiance. Appl Opt 13: 2160-2163. DOI: 10.1364/A0.13.002160.

Baluja KL, Zeippen CJ. 1988. M1 and E2 transition probabilities for states within the $2 \mathrm{p} 4$ configuration of the $\mathrm{O}$ I isoelectronic sequence. J Phys B: At Mol Phys 21: 1455-1471. DOI: 10.1088/ 0953-4075/21/9/007.

Bilitza D, Brown SA, Wang MY, Souza JR, Roddy PA. 2012. Measurements and IRI model predictions during the recent solar minimum. J Atmos Sol Terr Phys 86: 99-106. DOI: 10.1016/ j.jastp.2012.06.010.

Birn J,Artemyev AV, Baker DN, Echim M, Hoshino M, Zelenyi LM. 2012. Particle Acceleration in the Magneto tail and Aurora. Space Sci Rev 173: 49-102. DOI: 10.1007/s11214-012-9874-4.

Broadfoot AL, Hatfield DB, Anderson ER, Stone TC, Sandel BR, Gardner JA, Murad E, Knecht DJ, Pike CP, Viereck RA. 1997. N2 triplet band systems and atomic oxygen in the dayglow. $J$ Geophys Res 102: 11567-11584. DOI: 10.1029/97JA00771.

Christensen AB, Paxton LJ, Avery S, Craven J, Crowley G. 2003. Initial observations with the Global Ultraviolet Imager (GUVI) in the NASA TIMED satellite mission. J Geophys Res (Space Phys) 108: 1451. DOI: 10.1029/2003JA009918.

Cisek M, Makuch P, Petelski T. 2017. Comparison of meteorological conditions in Svalbard fjords: Hornsund and Kongsfjorden. Oceanologia 59(4): 413-421. DOI: https://doi.org/10.1016/ j.oceano.2017.06.004, URLhttp://www.sciencedirect.com/science/ article/pii/SQQ783234173QS672.

Diard T, de la Barriere F, Ferrec Y,Guerineau N, Rommeluere S, Le Coarer E, Martin G. 2016. Compact high-resolution microspectrometer on chip: spectral calibration and first spectrum. In: Micro- and Nanotechnology Sensors, Systems, and Applications VIII, vol. 9836 of Proc. SPIE, 98362W. DOI: 10.1117/12.2223692

Germany GA, Spann JF, Parks GK, Brittnacher MJ, Elsen R, Chen L, Lummerzheim D, Rees MH. 1998. Auroral Observations from the POLAR Ultraviolet Imager (UVI). Washington DC American Geophysical Union Geophysical Monograph Series 104: 149. DOI: $10.1029 / \mathrm{GM} 104 \mathrm{p} 0149$.
Gillard F, Ferrec Y, Guérineau N, Rommeluère S, Taboury J, Chavel P. 2012. Angular acceptance analysis of an infrared focal plane array with a built-in stationary Fourier transform spectrometer. J Opt Soc Am A 29: 936. DOI: 10.1364/JOSAA.29.000936.

Goguen JD, Stone TC, Kieffer HH, Buratti BJ. 2010. A new look at photometry of the Moon. Icarus 208: 548-557. DOI: 10.1016/ j.icarus.2010.03.025.

Grubbs G, Michell R, Samara M, Hampton D, Jahn J-M. 2016. A synthesis of star calibration techniques for ground-based narrowband electron-multiplying charge-coupled device imagers used in auroral photometry. J Geophys Res (Space Phys) 121: 5991-6002. DOI: 10.1002/2015JA022186.

Hecht JH, Strickland DJ, Conde MG. 2006. The application of ground-based optical techniques for inferring electron energy deposition and composition change during auroral precipitation events. Journal of Atmospheric and Solar-Terrestrial Physics 68: 1502-1519. DOI: 10.1016/j.jastp.2005.06.022.

Hevner R, Holemans W. 2011. An Advanced Standard for CubeSats, Paper SSC11-II-3. In: 25th Annual AIAA/USU Conference on Small Satellites, SSC11-II-3.

Kaeppler SR, Hampton DL, Nicolls MJ, Str0mme A, Solomon SC, Hecht JH, Conde MG. 2015. An investigation comparing groundbased techniques that quantify auroral electron flux and conductance. J Geophys Res (Space Phys) 120: 9038-9056. DOI: 10.1002/2015JA021396.

Kalegaev VV, Barinova WO, Myagkova IN, Eremeev VE, Parunakyan DA, Nguyen MD, Barinov OG. 2018. Empirical model of the high-latitude boundary of the Earth's outer radiation belt at altitudes of up to $1000 \mathrm{~km}$. Cosm. Res. 56(1): 32-37. DOI: $10.1134 / \mathrm{S} 0010952518010069$.

Kauristie K, Pulkkinen TI, Amm O, Viljanen A, Syrjasuo M, et al. 2001. Ground-based and satellite observations of high-latitude auroral activity in the dusk sector of the auroral oval. Ann Geophys 19: 1683-1696. DOI: 10.5194/angeo-19-1683-2001.

Kieffer HH. 1997. Photometric Stability of the Lunar Surface. Icarus 130(2): 323-327. DOI: https://doi.org/10.1006/icar.1997.5822.

Lacherade S, Aznay O, Fougnie B, Lebegue L. 2014. POLO: a unique dataset to derive the phase angle dependence of the Moon irradiance. In: Sensors, Systems, and Next-Generation Satellites XVIII, vol. 9241 of Proc. SPIE, 924112. DOI: 10.1117/12.2067283.

Le Coarer E, Guerineau N, Martin G, Rommeluere S, Ferrec Y, Schmitt B. 2014. SWIFTS-LA: an unprecedently small static imaging Fourier transform spectrometer. In: Proc. SPIE 10563, International Conference on Space Optics - ICSO 2014, 105634J, 17 November 2017. DOI: http://dx.doi.org/10.1117/12.2304159

Lilensten J, Blelly PL. 2002. The TEC and F2 parameters as tracers of the ionosphere and thermosphere. J Atmos Sol Terr Phys 64: 775-793. DOI: 10.1016/S1364-6826(02)00079-2.

Lilensten J, Moen J, Barthelemy M, Thissen R, Simon C, Lorentzen DA, Dutuit O, Amblard PO, Sigernes F. 2008. Polarization in aurorae: A new dimension for space environments studies. Geophys Res Lett 35: L08804. DOI: 10.1029/2007GL033006.

Lummerzheim D, Lilensten J. 1994. Electron transport and energy degradation in the ionosphere: Evaluation of the numerical solution, comparison with laboratory experiments and auroral observations. Ann Geophys 12: 1039-1051. DOI: 10.1007/ s00585-994-1039-7.

Megan-Gillies D, Knudsen D, Donovan E, Jackel B, Gillies R, Spanswick E. 2017. Identifying the $630 \mathrm{~nm}$ auroral arc emission height: A comparison of the triangulation, FAC profile, and electron density methods. J Geophys Res (Space Phys) 122: 8181-8197. DOI: 10.1002/2016JA023758. 
Newell PT, Liou K, Zhang Y, Sotirelis T, Paxton LJ, Mitchell EJ. 2014. OVATION Prime-2013: Extension of auroral precipitation model to higher disturbance levels. Space Weather 12: 368-379. DOI: $10.1002 / 2014$ SW001056.

Panasyuk MI, Podzolko MV, Kovtyukh AS, Brilkov IA, Vlasova NA, Kalegaev VV, Osedlo VI, Tulupov VI, Yashin IV. 2017. Optimization of measurements of the Earth's radiation belt particle fluxes. Cosm. Res. 55: 79-87. DOI: 10.1134/S0010952516060071.

Picone JM, Hedin AE, Drob DP, Aikin AC. 2002. NRLMSISE-00 empirical model of the atmosphere: Statistical comparisons and scientific issues. J Geophys Res (Space Phys) 107: 1468. DOI: 10.1029/2002JA009430.

Rommeluere S, Guerineau N, Haidar R, Deschamps J, de Borniol E, Million A, Chamonal J-P. 2008. Chamonal, and Destefanis. Infrared focal plane array with a built-in stationary Fouriertransform spectrometer: basic concepts.Opt Lett 3(3): 1062. DOI: 10.1364/OL.33.001062.

Saito H, Hirahara M, Mizuno T, Fukuda S, Fukushima Y, et al. 2011. Small satellite REIMEI for auroral observations.Acta Astronaut 69(7): 499-513. DOI: https://doi.org/10.1016/j.actaastro.2011.05. 007.

Sheese PE, Llewellyn EJ, Gattinger RL, Strong K. 2014. OH Meinel band nightglow profiles from OSIRIS observations. J Geophys Res (Atmos.) 119: 11. DOI: 10.1002/2014JD021617.

Sigernes F, Dyrland M, Brekke P, Chernouss S, Lorentzen DA, Oksavik K, Sterling Deehr C. 2011. Two methods to forecast auroral displays. J Space Weather Space Clim 1(27): A03, DOI: $10.1051 / \mathrm{swsc} / 2011003$.

Simon Wedlund C, Lamy H, Gustavsson B, Sergienko T, Brändström U. 2013. Estimating energy spectra of electron precipitation above auroral arcs from ground-based observations with radar and optics. J Geophys Res (Space Phys) 118: 3672-3691. DOI: 10.1002/jgra.50347.

Sims G, Ashley MCB, Cui X, Everett JR, Feng L, et al. 2012. Airglow and Aurorae at Dome A Antarctica. PASP 124: 637. DOI: $10.1086 / 666861$.
Stone TC, Kieffer HH. 2004. Assessment of uncertainty in ROLO lunar irradiance for on-orbit calibration Barnes WL, Butler JJ (Eds.). Earth Observing Systems IX, vol. 5542 of Proc. SPIE, 300-310. DOI: $10.1117 / 12.560236$

Strickland DJ, Meier RR, Hecht JH, Christensen AB. 1989. Deducing composition and incident electron spectra from ground-based auroral optical measurements. I - Theory and model results. II - A study of auroral red line processes. III Variations in oxygen density. J. Geophys. Res. 94: 13527-13539. DOI: 10.1029/JA094iA10p13527.

Teillet PM, Barsi JA, Chander G, Thome KJ. 2007. Prime candidate Earth targets for the post-launch radiometric calibration of spacebased optical imaging instruments. In Earth Observing Systems XIIIn: Earth Observing Systems XII. vol. 6677 of Proc. SPIE, 66770S. DOI: $10.1117 / 12.733156$

Torr MR, Torr DG. 1982. The role of metastable species in the thermosphere. Rev Geophys Space Phys 20: 91-144.

Tuttle S, Gustavsson B, Lanchester B. 2014. Temporal and spatial evolution of auroral electron energy spectra in a region surrounding the magnetic zenith. J Geophys Res (Space Phys) 119: 2318-2327. DOI: 10.1002/2013JA019627

Vialatte A, Barthelemy M, Lilensten J. 2017. Impact of Energetic Electron Precipitation on the Upper Atmosphere: Nitric Monoxide. Open Atmos Sci J 11: 88-104. DOI: 10.2174/ 1874282301711010088

Wagner SC, Hewison T, Stone T, Lacherade S, Fougnie B, Xiong X. 2015. A summary of the joint GSICS - CEOS/IVOS lunar calibration workshop: moving towards intercalibration using the Moon as a transfer target. In: Sensors, Systems, and NextGeneration Satellites XIX, vol. 9639 of Proc. SPIE, 96390Z. DOI: 10.1117/12.2193161.

Xiong X, Lacherade S, Lebegue L, Fougnie B, Angal A, Wang Z, Aznay O. 2014. Comparison of MODIS and PLEIADES Lunar observations. In: Sensors, Systems, and Next-Generation Satellites XVIII, vol. 9241 of Proc. SPIE, 924111. DOI: 10.1117/ 12.2067442 .

Cite this article as: Barthelemy M, Kalegaev V, Vialatte A, Le Coarer E, Kerstel E, et al. 2018. AMICal Sat and ATISE: two space missions for auroral monitoring. J. Space Weather Space Clim. 8, A44. 